Check for updates

Cite this: RSC Adv., 2018, 8, 15290

\title{
Enhancing performance of Ag-ZnO-Ag UV photodetector by piezo-phototronic effect $\uparrow$
}

Received 7th February 2018

Accepted 12th April 2018

DOI: $10.1039 / \mathrm{c} 8 \mathrm{ra01189c}$

rsc.li/rsc-advances

\author{
Xiaotong Zhang, ${ }^{a}$ Yu Qiu, (D) *ac Dechao Yang, ${ }^{\text {b }}$ Bing Li, ${ }^{\text {a }}$ Heqiu Zhang ${ }^{\text {ac }}$ \\ and Lizhong $\mathrm{Hu}^{\star \mathrm{ac}}$
}

In this work, an ultraviolet (UV) photodetector based on a ZnO nanowires (NWs) array with metalsemiconductor-metal Schottky junction structure was successfully fabricated on a flexible polyester fibre substrate by a low-temperature hydrothermal method. Subjected to a $0.2 \%$ tensile strain at $-1 \mathrm{~V}$, the $I_{\text {light }}$ and sensitivity of the as-prepared UV photodetector are lifted by $82 \%$ and $130 \%$, respectively. Furthermore, the response speed and recovery speed are significantly raised under the same tensile strain. The working principle can be explained as that the Schottky barrier height (SBH) is effectively improved by the negative strain-induced polarization at the metal-ZnO interface which is favorable for the separation of photogenerated electron-hole pairs. This work not only provides a facile and promising means to optimize the performance of a ZnO based MSM photodetector by applying a tensile strain but also opens up the way for fabrication and integration of $\mathrm{ZnO}$ photodetectors on flexible polyester fiber substrates.

\section{Introduction}

Recently, ultraviolet photodetectors depending on diverse materials and mechanisms have been widely discussed in many fields such as solar-blind detectors, environment monitoring and chemical analysis. ${ }^{1-3}$ Among a variety of candidates, $\mathrm{SiC},{ }^{\mathbf{4}}$ $\mathrm{GaN}^{5,6}$ and diamond ${ }^{7}$ are common semiconductors used for fabricating UV photodetectors. Compared with them, as a wide direct band-gap (3.37 eV) semiconductor, $\mathrm{ZnO}$ owns some unique merits to serve as a photo-absorber layer in a UV photodetector, for example, low price, simple synthesizing process, ${ }^{8-10}$ high response speed and abundant 1-D nanostructures. Hence, $\mathrm{ZnO}$ has attracted a great deal of interest and possesses the potential to be an alternative material in UV detectors. ${ }^{11-16}$ To improve the performance of $\mathrm{ZnO}$ based UV detectors, many materials such as PEDOT: PSS, ${ }^{17} \mathrm{Ag},{ }^{18,19} \mathrm{ZnS},{ }^{20,21}$ $\mathrm{NiO}^{22,23}$ have been employed to form a hetero-junction with ZnO. In terms of recent research, MSM structured Schottky junction UV detector formed by $\mathrm{Ag}$ and $\mathrm{ZnO}$ is extremely facile to be prepared which not only increases the photoresponsivity but also restricts the dark current. The low-cost, uncomplicated

${ }^{a}$ School of Physics, Dalian University of Technology, Dalian 116024, People's Republic of China.E-mail: yuqiu@dlut.edu.cn

${ }^{b}$ Department of Electronic Engineering, Dalian Neusoft University of Information, Dalian, 116024, People's Republic of China

${ }^{c}$ The Key Laboratory for Micro/Nano Technology and System of Liaoning Province, Dalian University of Technology, Dalian 116024, People's Republic of China

$\dagger$ Electronic supplementary information (ESI) available. See DOI: 10.1039/c8ra01189c fabricating process supplies a possibility for realization of largescale integration and mass production.

$\mathrm{ZnO}$, a wurtzite-structured semiconductor with non-central symmetry, will generate a piezoelectric polarization under an external force. Based on the piezoelectric, semiconductor, phototronic three-way coupling characteristic, plenty of electronic devices have been developed including solar cells, ${ }^{24}$ light emitted diodes, ${ }^{25}$ photodetectors, ${ }^{20,26}$ strain sensors, ${ }^{27}$ field effect transistors ${ }^{28}$ and so on. For these junction-based devices, the transport, separation, recombination of charge carriers, barrier height and build-in electric field at the interface are modulated by strain-induced piezoelectric polarization. ${ }^{27}$ In respect of an $\mathrm{Ag}-\mathrm{ZnO}$ Schottky junction UV photodetector, the depletion region at MS contact area provides a strong build-in field to separate electron-hole pairs under illumination. Therefore, according to the piezo-phototronics theory, the performance of the UV photodetector can be monitored and strengthened by external strain. ${ }^{29}$

Conventional UV photodetectors are usually manufactured on hard substrates like silicon, sapphire, ITO glass and all kinds of metal foil. With the revolution of science and technology, soft portable electronic devices which remarkably facilitate people's life have shown large scientific potential and commercial perspective. $^{\mathbf{3 0 , 3 1}}$ Therefore, it is a surge demand to develop electronic devices based on flexible, portable, wearable substrates. Recently, diverse soft substrates (like paper, flexible plastic and polymers) have been gradually applicated in variety of electronic devices, but these substrates also suffer from certain defects and need further research. For example, paperbased electronic devices are not durable enough and crispy. 
The plastic-based electronic devices are hard to integrate with wearable materials such as fiber and cloth. To our best knowledge, the fabrication of opto-electronic devices on fabric or fabric-like substrates is rarely reported. Therefore, the study about fabrication of components on fabric substrates can be novel and significant which may promote the development and integration of devices on wearable substrates.

In this paper, a ZnO MSM UV photodetector has been successfully synthesized on the flexible polyester fibre substrate by a low-temperature hydrothermal process. The detailed morphology and structural characterization are shown in the paper. More importantly, the UV photodetector can be further improved by piezo-phototronic effect under a small external strain. Basic mechanisms for observed characteristics are discussed in detail. This work not only studies the impact of deformation towards performance of Schottky-junction photodetector but also provides an effective method to optimize $\mathrm{ZnO}$ based MSM UV photodetector by a tensile strain. Furthermore, our work also broadens the road for the fabrication of photodetectors on flexible polyester fibers.

\section{Experimental}

\subsection{Synthesis of ZnO NWs on polyester fibre substrate}

For the construction of $\mathrm{ZnO}$ UV photodetector, we adopted commercially available polyester fibre as the substrate, which is a quite common material used everywhere in our daily life. Polyester fibre is born with massive merits serving as a substrate. For example, it is light-weight, wearable, portable, biocompatible and cheap. ${ }^{32}$ Firstly, the as-prepared substrates are cut into $1 \mathrm{~cm} \times 1 \mathrm{~cm}$ pieces. Then, the pre-treated substrates are cleaned ultrasonically in turn by acetone, ethanol and deionized water for 15 minutes, respectively. After dried in heating oven for 1 hour at $80{ }^{\circ} \mathrm{C}$, the polyester fiber substrates are well prepared. Before the growth of $\mathrm{ZnO}$ NWs, a $20 \mathrm{~nm}$-thick $\mathrm{ZnO}$ seed layer is deposited on the polyester substrates with room temperature radio frequency magnetron sputtering. The background pressure of the vacuum chamber is $3 \times 10^{-4} \mathrm{~Pa}$ and the pressure of the sputtering gas argon is 3.5 $\mathrm{Pa}$. The radio frequency power is $140 \mathrm{~W}$. Next, ZnO NWs are perpendicularly synthesized on the seed layer by hydrothermal method. The aqueous solution is composed of $30 \mathrm{mmol} \mathrm{L}^{-1}$ zinc acetate dehydrate and hexamethylenetetramine (molar ratio $1: 1)$. Then, the seed layer substrates are kept vertically in a Teflon reaction kettle filled with solution and heated at $95{ }^{\circ} \mathrm{C}$ for 3 hours. After the reaction, the samples are rinsed for several times by deionized water and completely dried in heating oven.

\subsection{Fabrication of $\mathrm{Ag}-\mathrm{ZnO}-\mathrm{Ag} \mathrm{UV}$ photodetector}

For the fabrication of $\mathrm{ZnO} \mathrm{UV}$ photodetector, the ZnO NWs substrate is coated with two thin layers of $\mathrm{Ag}$ paste onto two ends of substrates to serve as electrodes. The distance between two electrodes is about $0.5 \mathrm{~cm}$ and the effective working area is about $0.5 \times 1 \mathrm{~cm}^{2}$. Then both of $\mathrm{Ag}$ electrodes are connected with two $\mathrm{Cu}$ testing wires and dried in oven at $85{ }^{\circ} \mathrm{C}$ for half an hour. Finally, the whole device is successfully prepared.

\subsection{Characterization}

The crystal structure and surface morphology of ZnO NWs array are characterized by X-ray diffraction (XRD) and scanning electron microscope (SEM). Room temperature photoluminescence (PL) with a $325 \mathrm{~nm} \mathrm{He-Cd} \mathrm{laser} \mathrm{is} \mathrm{carried} \mathrm{out} \mathrm{to}$ perform the crystallographic quality of ZnO NWs. A Keithley 4200 semiconductor characterization system is used to measure photoelectric properties of the photodetector under a UV (365 $\mathrm{nm})$ illumination $(17 \mathrm{~mW})$.

\section{Results and discussion}

\subsection{Surface morphology and crystallinity}

The schematic diagram of the whole growth process is shown in Fig. 1, including ZnO seed layer deposition, ZnO NWs growth and conductive electrode preparation. The morphology images of ZnO NWs grown on the polyester fiber substrate under different magnifications are shown in Fig. 2(a-c). From Fig. 2(a) and (b), we can see that ZnO NWs are uniformly and densely covered onto polyester fibers. As shown in Fig. 2(c), all the ZnO NWs are in hexagonal prismatic structure with a diameter of 200-300 nm and a length of 4-5 $\mu \mathrm{m}$. The well-aligned 1-D NWs have a clean and smooth top and side surfaces which not only contributes to the absorption of incident light from all directions but also is in favor of the formation of MSM Schottky junction.

The XRD patterns of ZnO NWs are utilized to characterize the crystal phase. As presented in Fig. 2(d), the (002), (100), (101) diffraction peaks are clearly observed which proves that the $\mathrm{ZnO}$ NWs have a hexagonal wurtzite structure and grow along $c$-axis. The XRD patterns also demonstrate the ZnO synthesized on polyester fibers exhibits a perfect crystalline quality and lays a good foundation for the performance of devices. The picture inside Fig. 2(d) indicated that the device is highly flexible and can be bent and fully recover. Fig. S1† also displays the roomtemperature photoluminescence spectrum of prepared $\mathrm{ZnO}$ NWs sample. A near band edge (NBE) emission at $360 \mathrm{~nm}$ is obviously observed which results from the inter-band radiation combination of free excitons. Besides that, the broad, deeplevel, yellow emissions also exist between $550-650 \mathrm{~nm}$ due to (a)

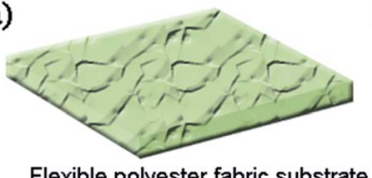

Flexible polyester fabric substrate

(c)

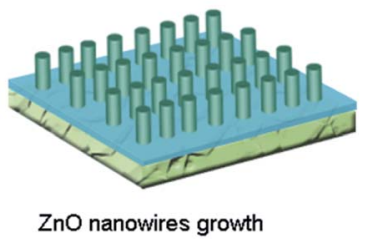

(b)

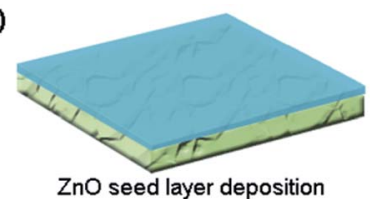

(d)

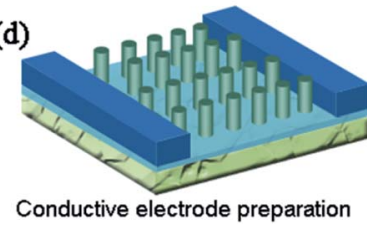

Fig. 1 Schematic illustration of fabricating processes of $\mathrm{Ag}-\mathrm{ZnO}-\mathrm{Ag}$ photodetector: (a) polyester fabric substrate preparation, (b) ZnO seed layer deposition, (c) ZnO NWs growth, (d) conductive electrode preparation. 

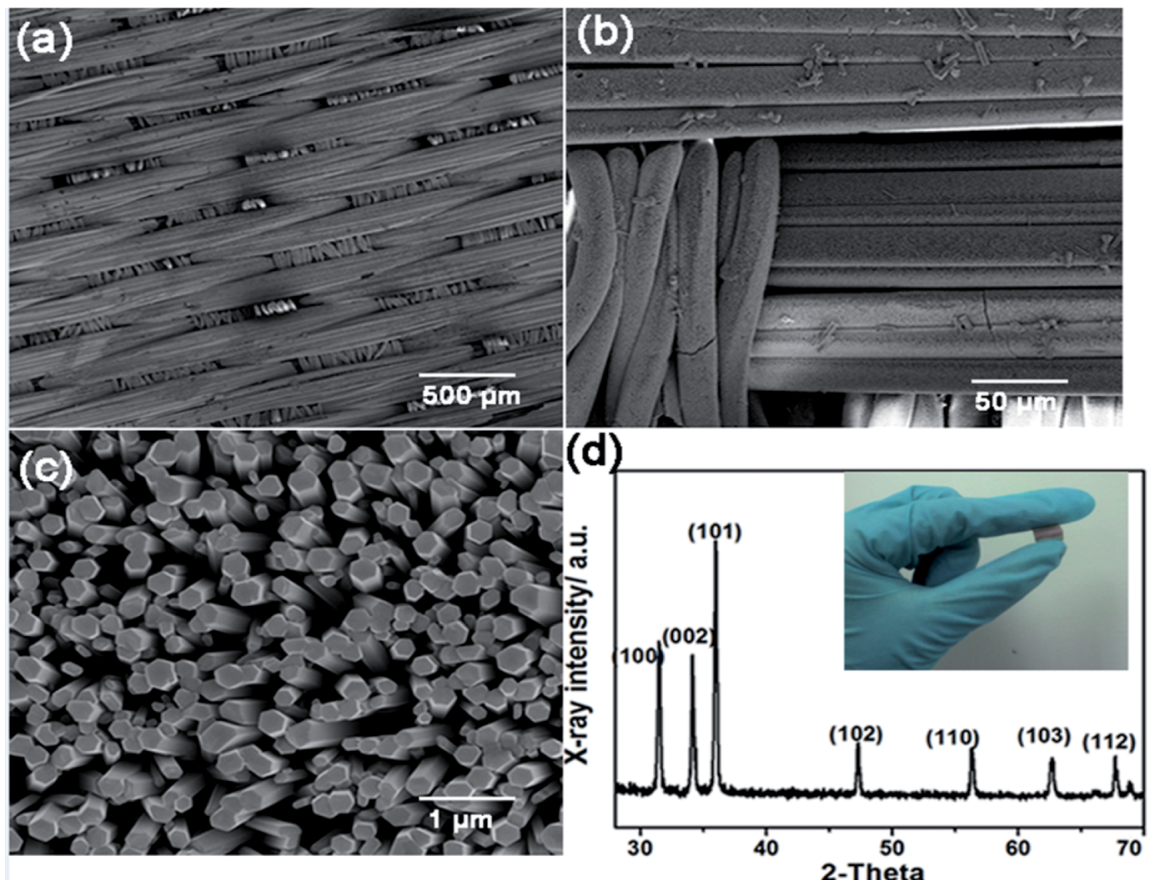

Fig. 2 (a) and (b) Low-magnification SEM image of ZnO NWs on polyester fibre substrate. (c) High-magnification SEM image of ZnO NWs. (d) XRD pattern of $\mathrm{ZnO} N W s$ and optical image of as-fabricated device (inset).

the impurity and defects of $\mathrm{ZnO}$ NWs such as oxygen vacancies. It should be noted that $\mathrm{ZnO}$ has high-density surface states which can lead to the Fermi-level Pinning Effect and surface band-bending phenomenon and the decrease of carriers' concentration. According to the theory mentioned above, although electron affinities of $\mathrm{Ag}$ and $\mathrm{ZnO}$ are nearly close, they can still compose a Schottky junction barrier. The barrier height is mainly dominated by the surface states rather than the work function. ${ }^{28}$

\subsection{The performance of photodetector under UV illumination}

To study the impact of piezo-phototronic effect on UV photodetector, a measuring setup is established. As shown in Fig. S2, $\dagger$ a plastic board (PB) which is about $20 \mathrm{~cm}$ long is fixed to a fixed sample platform at one edge and another edge is connected with a 3D portable mechanical stage. The PB is elastic and can be bent and curved with the movement of $3 \mathrm{D}$ mechanical stage. Our sample is mounted on the center of PB while the $365 \mathrm{~nm}$ UV illuminant is exactly above it. Considering that the device is much smaller than the $\mathrm{PB}$, shifting the $3 \mathrm{D}$ mechanical stage down for $1 \mathrm{~cm}$ equals to subjecting a tensile strain to the photodetector. ${ }^{29,30}$ On the contrast, when shifting up the 3D stage, the photodetector receives a compressive strain. The deformation of photodetector $\varepsilon$ can be calculated from $\varepsilon=d / 2 R$, where $d$ is the up/down moving distance of 3D stage, $R$ is the bending curvature. In this paper, while $d$ is $1 \mathrm{~cm}$ and the length of $\mathrm{PB}$ is $20 \mathrm{~cm}$, according to the formulation $h=2 R \sin ^{2}(\theta / 2)=$ $2 R \sin ^{2}(L / 2 R)$, the strain $\varepsilon$ was calculated to be $0.2 \%$.

Fig. 3(a) shows typical the $I-V$ characteristic of $\mathrm{Ag}-\mathrm{ZnO}-\mathrm{Ag}$ UV photodetector on flexible polyester fibre substrate in the dark (black) and under UV illumination ( $365 \mathrm{~nm}, 0.5 \mu \mathrm{W} \mathrm{cm}{ }^{-2}$ ). The absolute current is improved from $25 \mathrm{nA}$ (dark current) to $150 \mathrm{nA}$ at a bias of $5 \mathrm{~V}$ meanwhile the photocurrent is about 6 times higher than the dark current. The sensitivity which is defined as $\left(I_{\text {light }}-I_{\text {dark }}\right) / I_{\text {dark }}$ is as much as 5 here. Fig. 3(b) depicts the photocurrent of the as-fabricated $\mathrm{ZnO}$ UV photodetector under illumination at a bias of $-4 \mathrm{~V}$. The photocurrent-time curve demonstrates that the device has a rapid response speed and a fast recovery speed. Moreover, after many times' repeated cycles, the photocurrent doesn't drift and can fully recover which proves our sensors have a good stability and repeatability.

The symmetric rectifying $I-V$ characteristics with and without illumination indicate that two back to back Schottky junctions based on $\mathrm{Ag}-\mathrm{ZnO}-\mathrm{Ag}$ structure have been successfully fabricated. On one hand, the MSM design can dramatically suppress the dark current thereby increasing the sensitivity; on the other hand it could monitor the separation and transport of carriers through the Schottky junction. When a constant voltage bias is applied on the photodetector, the voltage drop mainly locates at the reversely biased Schottky junction, ${ }^{33,34}$ therefore, the photoelectric property of the device is chiefly dominated by the reversely biased Schottky junction. Hence, in dark condition, the current should be expressed in terms of thermionicemission theory as follows:

$$
I=A A^{*} T^{2} \exp \left(\frac{-e \phi_{\mathrm{Bn}}}{k T}\right)\left[\exp \left(\frac{e V}{k T}\right)-1\right]
$$

where $A$ is the area of the contact, $A^{*}$ is Richardson constant, $T$ is the Kelvin temperature, $e$ is the charge of electron, $\phi_{\mathrm{Bn}}$ refers to the height of Schottky junction barrier, $k$ is the Boltzmann constant, $V$ represents the external bias. 

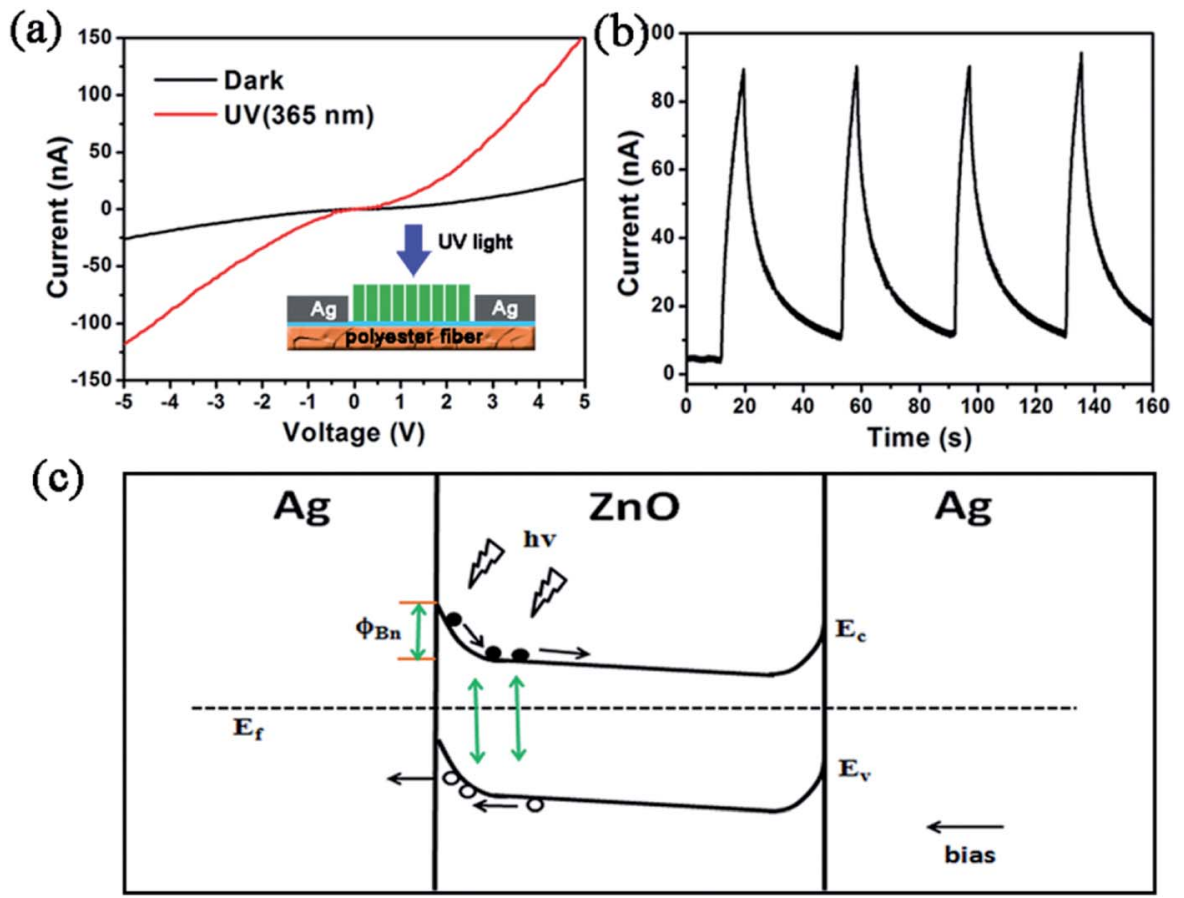

Fig. 3 (a) The $I-V$ characteristic curves in dark and under UV illumination. (b) The $I-T$ characteristic curves of the ZnO UV photodetector at a bias of $-4 \mathrm{~V}$. (c) Schematic energy band diagram for illustrating the working principle of the UV photodetector under no strain.

As to the photocurrent, the situation can be discussed in two aspects. Outside the contact area, NWs will absorb the oxygen molecules from atmosphere and the $\mathrm{O}_{2}$ will capture electrons from $\mathrm{ZnO}$ which brings about the formation of a large quantity of holes distributing among the surface in dark. Then the free holes near surface and the free electrons from inner part construct a depletion region which decreases the conductivity of ZnO NWs. ${ }^{35}$ When the detector is exposed to UV illumination whose energy is greater than the bandgap of $\mathrm{ZnO}$, electron-hole pairs are generated along the NWs. The photo-generated holes compensate with trapped electrons then oxygen molecules are desorbed into the air. At the same time, the unpaired photogenerated electrons are released into internal NWs and lift the conductivity of photodetector. ${ }^{36,37}$ When considering about the junction area, we have noticed that half proportion of the device belongs to the junction area and the characteristic of Schottky junction is relatively remarkable. As a consequence, according to the energy band schematic diagram in Fig. 3(c), when illumination falls on the sensor, electron-hole pairs are generated at two heterojunctions. At the reversely biased junction area, the generated holes flow to the $\mathrm{Ag}$ electrode and the generated electrons drift to the ZnO NWs array. ${ }^{15}$ Nevertheless, the photocurrent produced at forward biased Schottky junction is seriously confined by the reversely biased junction, so we won't take the current generated at forward biased junction into consideration. Then the photocurrent is created by the cooperated effect inside and outside the junction area. With regard to the phenomenon that the recovery time is longer than the response time presented in Fig. 3(b), the reason can be explained as that after the illumination is withdrawn, the $\mathrm{ZnO}$
NWs absorb oxygen molecules again followed by the decrease of conductivity. This process takes more time so the recovery speed is a bit lower.

\subsection{Piezoelectric effect on the performance of UV photodetector}

Fig. 4 has illustrated the influence of external strain on the performance of UV photodetector. The $I-V$ characteristics under tensile strain and compressive strain with and without UV light measured at a bias of $-5 \mathrm{~V}$ to $+5 \mathrm{~V}$ are described in Fig. 4 (a) and (b). As displayed in Fig. 4(a), at biases of $-1 \mathrm{~V},-2 \mathrm{~V}$ and $-3 \mathrm{~V}$ and under a $0.2 \%$ tensile strain, the absolute photocurrents increase from $44.3 \mathrm{nA}, 120.3 \mathrm{nA}, 221.1 \mathrm{nA}$ to $80.8 \mathrm{nA}, 196.5 \mathrm{nA}$, $331.9 \mathrm{nA}$ and the sensitivities improve from $1.72,1.47,1.29$ to $3.96,3.03,2.45$, respectively. On the contrary, from Fig. 4(b), we can see that both of the photocurrent and sensitivity decline under compressive strain. For example, at a bias of $-3 \mathrm{~V}$, the absolute photocurrent and sensitivity reduce from $211.2 \mathrm{nA}$ and 1.49 to $173.5 \mathrm{nA}$ and 1.05 as a function of a $0.2 \%$ compressive strain. In addition, the photocurrent-time curves at a bias of $-1.5 \mathrm{~V}$ under no-strain condition and under a $0.2 \%$ tensile strain are depicted in Fig. 4(c) and (d). As shown in the diagram, the average peak photocurrent is lifted from $92 \mathrm{nA}$ to $153 \mathrm{nA}$ under the tensile strain. Moreover, the average time of photocurrent responding to peak value and the average time of photocurrent recovering to initial value are decreased from $11.3 \mathrm{~s}$ and $18.8 \mathrm{~s}$ to $4.4 \mathrm{~s}$ and $1.9 \mathrm{~s}$. Through comparing two $I-T$ characteristics with and without tensile strain, a conclusion has been summarized that tensile strain can not only improve the 

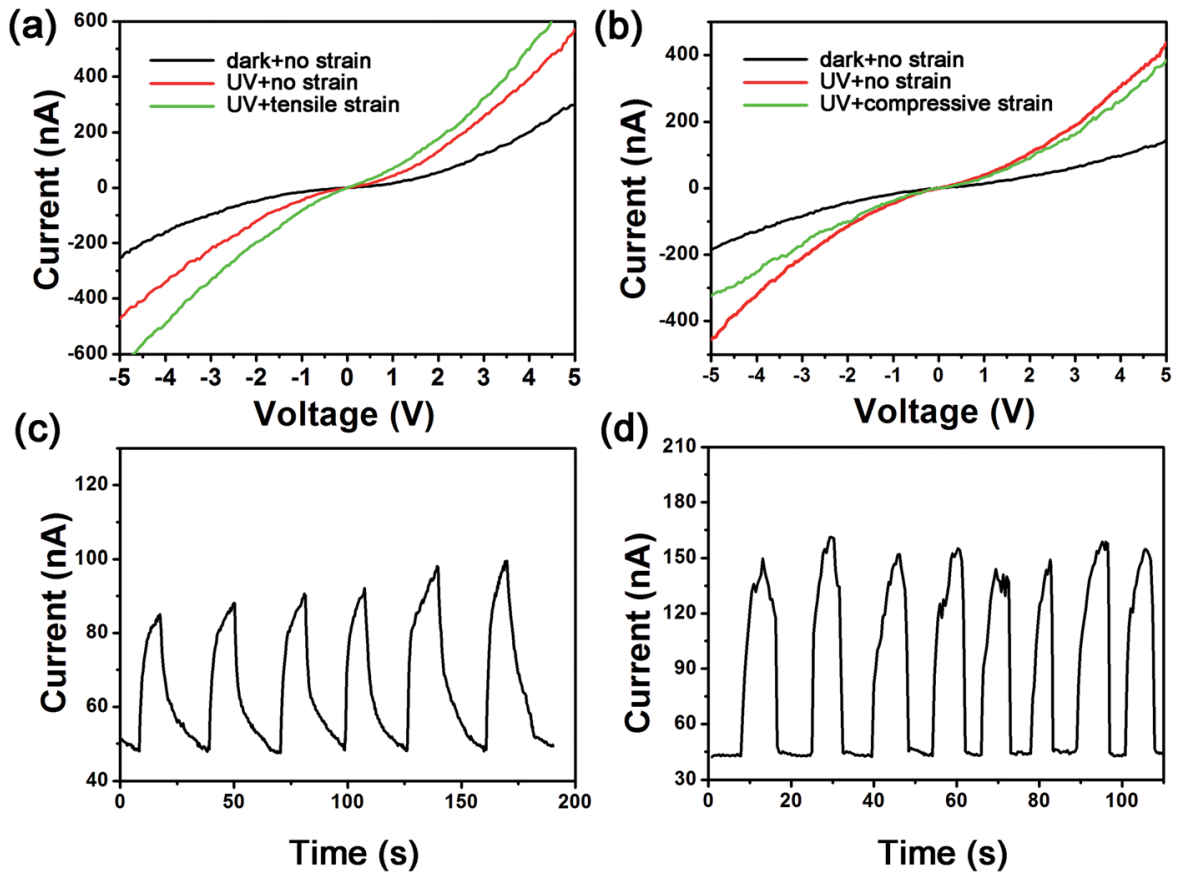

Fig. 4 (a) The I-V characteristic curves with/without tensile strain and with/without UV light. (b) The I-V characteristic curves with/without compressive strain and with/without UV light. (c) Time dependence of the photocurrent without external strain at a bias of $-1.5 \mathrm{~V}$. (d) Time dependence of the photocurrent with tensile strain at a bias of $-1.5 \mathrm{~V}$.

photocurrent but also promote the response speed and recovery speed.

The enhancing performance of $\mathrm{Ag}-\mathrm{ZnO}-\mathrm{Ag}$ UV photodetector by piezo-phototronic effect can be explained from the principle proposed in Fig. 5. Fig. 5(a) and (b) are schematic diagrams of energy band with tensile strain and compressive strain under UV illumination. When a tensile strain is applied to the device, a negative piezo-polarization will be formed at the interface of $\mathrm{Ag}$ and $\mathrm{ZnO}$. The strain-induced negative piezopolarization charges gathering at the junction area elevate the Schottky barrier height (SBH) as shown in Fig. 5(a) and the variation of $\mathrm{SBH}$ caused by piezoelectric effect can be simplified as: ${ }^{38}$

$$
\Delta \phi_{\text {piezo }}=-\frac{q^{2}}{2 \varepsilon_{\mathrm{s}}} \rho_{\text {piezo }} W_{\text {piezo }}^{2}
$$

where $\varepsilon_{\mathrm{s}}$ is relative dielectric constant, $q$ is the charge of the electron, $\rho_{\text {piezo }}$ represents the density of piezoelectric charges and $W_{\text {piezo }}$ refers to the width of piezo-charges. In addition, under the tensile strain, the width of depletion region is broadened as well on account of the negative piezo-charges and can be described as following:

$$
W=\left[\frac{2 \varepsilon_{\mathrm{ZnO}}\left(\phi_{\mathrm{Bn}}+V_{\mathrm{R}}\right)}{e N_{\mathrm{d}}}\right]^{\frac{1}{2}}
$$

where $\varepsilon_{\mathrm{ZnO}}$ is the dielectric constant of $\mathrm{ZnO}, V_{\mathrm{R}}$ is the reversely voltage bias, $\phi_{\mathrm{Bn}}$ is the Schottky junction barrier height that can be enhanced by external strain and $N_{\mathrm{d}}$ is the density of the electrons in ZnO. As illustrated in Fig. 5(a), depletion width is broadened from $W_{0}$ to $W_{\mathrm{pz}}$ which increases monotonically with the SBH.

$$
E=-\frac{e N_{\mathrm{d}}}{\varepsilon_{\mathrm{ZnO}}}(W-x), \quad 0 \leq x \leq W
$$

Therefore, from eqn (4) we can conclude that the build-in electric field is strengthened by the enhanced SBH and depletion width. Overall, the enhanced Schottky barrier height, depletion region width, and build-in field are beneficial for the separation of photogenerated electron-hole pairs, thus a higher photocurrent, sensitivity and a rapider response, recovery speed are obtained. This is the principle of the $\mathrm{Ag}-\mathrm{ZnO}-\mathrm{Ag} \mathrm{UV}$

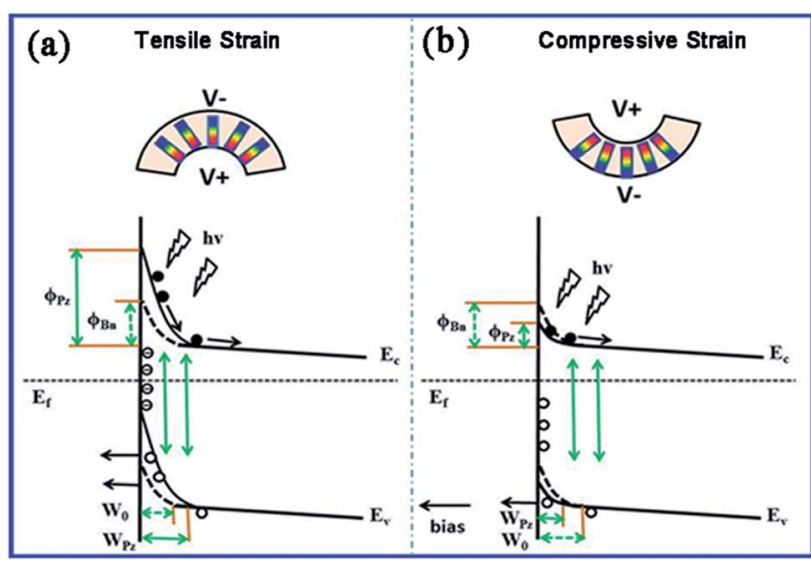

Fig. 5 Schematics and energy band diagrams for illustrating the working principle of the UV photodetector under (a) tensile strain and (b) compressive strain. 
photodetector enhancing performance by piezo-phototronic effect. Inversely, a positive piezo-polarization is induced at the $\mathrm{Ag} / \mathrm{ZnO}$ interface by compressive strain which decreases the SBH, depletion width, build-in field and weakens the separation of photogenerated electron-hole pairs. Then the photocurrent and sensitivity are reduced. This work also provides a promising approach to modulate the property of $\mathrm{ZnO}$ based photosensors and evidence the feasibility of flexible polyester fibers applicated in piezo-photoelectronic devices.

\section{Conclusion}

In summary, we have successfully fabricated the Ag-ZnO-Ag UV photodetector based on ZnO NWs by a simple low-temperature hydrothermal method. On account of the unique piezosemiconductor property of $\mathrm{ZnO}$, the performance of $\mathrm{UV}$ photodetector can be optimized by an external strain. Under a $0.2 \%$ tensile strain, the photocurrent and sensitivity are raised by $82 \%$ and $130 \%$ at a bias of $-1 \mathrm{~V}$. Moreover, at a bias of $-1.5 \mathrm{~V}$, the response speed and recovery speed are significantly improved at the same strain. The result shows that the negative piezo-polarization charges induced by tensile strain increase the Schottky junction barrier height, depletion region width and build-in electric field, which is favorable for the separation of photogenerated electron-hole pairs. This work broadens the road for integration of electronic devices on flexible polyester fibers and offers a facile means to enhance the performance of $\mathrm{ZnO}$ based photodetectors by piezo-phototronic effect.

\section{Conflicts of interest}

There are no conflicts to declare.

\section{Acknowledgements}

This work was supported by the NSFC (Project No. 61504018), Support high-level innovative entrepreneurial talent project in Dalian (2015R094), the Doctoral Scientific Research Foundation of Liaoning Province (Project No. 201501193), General Project of Scientific Research of the Education Department of Liaoning Province (L2015040), and Foundation of Key laboratory for Micro/Nano Technology and System of Liaoning Province (20140405).

\section{References}

1 W. D. Li and S. Y. Chou, Solar-blind deep-UV band-pass filter (250-350 nm) consisting of a metal nano-grid fabricated by nanoimprint lithography, Opt. Express, 2010, 18(2), 931-937.

2 M. Razeghi and A. Rogalski, Semiconductor ultraviolet detectors, J. Appl. Phys., 1996, 79(10), 7433-7473.

3 E. Monroy, F. Omnès and F. Calle, Topical review: widebandgap semiconductor ultraviolet photodetectors, Semicond. Sci. Technol., 2003, 18(4), R33.

4 V. I. Sankin and V. P. Chelibanov, Silicon Carbide Ultraviolet Photodetectors and Their Application in Ecological Monitoring, Phys. Status Solidi, 2015, 185(1), 153-158.
5 N. Vanhove, J. John, A. Lorenz, K. Cheng, G. Borghs and J. E. M. Haverkort, ITON Schottky contacts for GaN based UV photodetectors, Appl. Surf. Sci., 2006, 253(5), 2930-2932.

6 A. V. Babichev, H. Zhang, P. Lavenus, F. H. Julien, A. Y. Egorov, Y. T. Lin, L. W. Tu and M. Tchernycheva, GaN nanowire ultraviolet photodetector with a graphene transparent contact, Appl. Phys. Lett., 2013, 103(20), 14311436.

7 Z. Liu, J. P. Ao, F. Li, W. Wang, J. Wang, J. Zhang and H. X. Wang, Fabrication of three dimensional diamond ultraviolet photodetector through down-top method, Appl. Phys. Lett., 2016, 109(15), 982.

8 S. Singh and P. Chakrabarti, Simulation, fabrication and characterization of $\mathrm{ZnO}$ based thin film transistors grown by radio frequency magnetron sputtering, Am. J. Nanosci. Nanotechnol., 2012, 12(3), 1880.

9 S. Singh, Al doped ZnO based metal-semiconductor-metal and metal-insulator-semiconductor-insulator-metal UV sensors, Optik, 2016, 127(7), 3523-3526.

$10 \mathrm{~S}$. Singh and S. Kalaivani, Improvement in the performance of AZO based MISIM photodetectors using a spray coated $\mathrm{Al}_{2} \mathrm{O}_{3}$ insulating layer, Optik, 2016, 127(12), 4984-4986.

$11 \mathrm{~S}$. Singh and P. Chakrabarti, Effect of mesa structure formation on the electrical properties of zinc oxide thin film transistors, Am. J. Nanosci. Nanotechnol., 2014, 14(5), 3552-3556.

12 B. Liu, J. Xu, S. Ran, Z. Wang, D. Chen and G. Shen, Highperformance photodetectors, photocatalysts, and gas sensors based on polyol reflux synthesized porous $\mathrm{ZnO}$ nanosheets, CrystEngComm, 2012, 14(14), 4582-4588.

13 W. Cheng, L. Tang, J. Xiang, R. Ji and J. Zhao, An extreme high-performance ultraviolet photovoltaic detector based on a $\mathrm{ZnO}$ nanorods/phenanthrene heterojunction, $R S C$ Adv., 2016, 6(15), 12076-12080.

14 A. A. Chaaya, M. Bechelany, S. Balme and P. Miele, ZnO 1D nanostructures designed by combining atomic layer deposition and electrospinning for UV sensor applications, J. Mater. Chem. A, 2014, 2(48), 20650-20658.

15 Z. Zhang, Q. Liao, Y. Yu, X. Wang and Y. Zhang, Enhanced photoresponse of $\mathrm{ZnO}$ nanorods-based self-powered photodetector by piezotronic interface engineering, Nano Energy, 2014, 9(16), 237-244.

16 X. Zhang, X. Han, J. Su, Q. Zhang and Y. Gao, Well vertically aligned ZnO nanowire arrays with an ultra-fast recovery time for UV photodetector, Appl. Phys. A: Mater. Sci. Process., 2012, 107(2), 255-260.

17 P. Lin, X. Yan, Z. Zhang, Y. Shen, Y. Zhao, Z. Bai and Y. Zhang, Self-powered UV photosensor based on PEDOT: $\mathrm{PSS} / \mathrm{ZnO}$ micro/nanowire with strain-modulated photoresponse, ACS Appl. Mater. Interfaces, 2013, 5(9), 3671-3676.

18 X. Zhao, F. Wang, L. Shi, Y. Wang, H. Zhao and D. Zhao, Performance enhancement in $\mathrm{ZnO}$ nanowire based double Schottky-Barriers photodetector by applying optimized Ag nanoparticles, RSC Adv., 2016, 6(6), 4634-4639.

19 Y. Liu, X. Zhang, J. Su, H. Li, Q. Zhang and Y. Gao, Ag nanoparticles@ZnO nanowire composite arrays: an 
absorption enhanced UV photodetector, Opt. Express, 2014, 22(24), 30148-30155.

20 S. C. Rai, K. Wang, Y. Ding, J. K. Marmon, M. Bhatt, Y. Zhang, W. Zhou and Z. L. Wang, Piezo-phototronic Effect Enhanced UV/Visible Photodetector Based on Fully Wide Band Gap Type-II $\mathrm{ZnO} / \mathrm{ZnS}$ Core/Shell Nanowire Array, ACS Nano, 2015, 9(6), 6419-6427.

21 X. Fang, Z. Wei, R. Chen, J. Tang, H. Zhao, L. Zhang, D. Zhao, D. Fang, J. Li and F. Fang, Influence of Exciton Localization on the Emission and Ultraviolet Photoresponse of $\mathrm{ZnO} / \mathrm{ZnS}$ Core-Shell Nanowires, ACS Appl. Mater. Interfaces, 2015, 7(19), 10331.

22 J. R. D. Retamal, C. Y. Chen, D. H. Lien, M. R. S. Huang, C. A. Lin, C. P. Liu and J. H. He, Concurrent Improvement in Photogain and Speed of a Metal Oxide Nanowire Photodetector through Enhancing Surface Band Bending via Incorporating a Nanoscale Heterojunction, ACS Photonics, 2014, 1(4), 354-359.

23 d. Y. Kim, J. Ryu, J. Manders, J. Lee and F. So, Air-stable, solution-processed oxide $\mathrm{p}-\mathrm{n}$ heterojunction ultraviolet photodetector, ACS Appl. Mater. Interfaces, 2014, 6(3), 13701374.

24 X. Wen, W. Wu and Z. L. Wang, Effective piezo-phototronic enhancement of solar cell performance by tuning material properties, Nano Energy, 2013, 2(6), 1093-1100.

25 Q. Yang, Y. Liu, C. Pan, J. Chen, X. Wen and Z. L. Wang, Largely enhanced efficiency in $\mathrm{ZnO}$ nanowire/p-polymer hybridized inorganic/organic ultraviolet light-emitting diode by piezo-phototronic effect, Nano Lett., 2013, 13(2), 607.

26 J. Liu, Z. Yang, C. Liu, M. Peng, A. Yu, J. Kou, L. Wei, J. Zhai and J. Liu, Piezo-phototronic effect enhanced UV photodetector based on $\mathrm{CuI} / \mathrm{ZnO}$ double-shell grown on flexible copper microwire, Nanoscale Res. Lett., 2016, 11(1), 281.

27 J. Shi, M. B. Starr and X. Wang, Band Structure Engineering at Heterojunction Interfaces via the Piezotronic Effect, $A d v$. Mater., 2012, 24(34), 4683.

28 M. Peng, Y. Liu, A. Yu, Y. Zhang, C. Liu, J. Liu, W. Wu, K. Zhang, X. Shi and J. Kou, Flexible Self-Powered GaN Ultraviolet Photoswitch with Piezo-Phototronic Effect Enhanced On/Off Ratio, ACS Nano, 2016, 10(1), 1572.
29 H. Li, X. Zhang, N. Liu, L. Ding, J. Tao, S. Wang, J. Su, L. Li and Y. Gao, Enhanced photo-response properties of a single $\mathrm{ZnO}$ microwire photodetector by coupling effect between localized Schottky barriers and piezoelectric potential, Opt. Express, 2015, 23(16), 21204.

30 S. Majumder, T. Mondal and M. J. Deen, Wearable Sensors for Remote Health Monitoring, Sensors, 2017, 17(1), 112350-12355.

31 A. Proto, M. Penhaker, D. Bibbo, D. Vala, S. Conforto and M. Schmid, Measurements of Generated Energy/Electrical Quantities from Locomotion Activities Using Piezoelectric Wearable Sensors for Body Motion Energy Harvesting, Sensors, 2016, 16(4), 524.

32 Y. Qiu, J. Lei, D. Yang, B. Yin, H. Zhang, J. Bian, J. Ji, Y. Liu, Y. Zhao and Y. Luo, Enhanced performance of wearable piezoelectric nanogenerator fabricated by two-step hydrothermal process, Appl. Phys. Lett., 2014, 104(11), 242.

33 Q. Liao, M. Mohr, X. Zhang, Z. Zhang, Y. Zhang and H. J. Fecht, Carbon fiber-ZnO nanowire hybrid structures for flexible and adaptable strain sensors, Nanoscale, 2013, 5(24), 12350.

34 Z. Wang, R. Yu, X. Wen, Y. Liu, C. Pan, W. Wu and Z. L. Wang, Optimizing performance of silicon-based $p-n$ junction photodetectors by the piezo-phototronic effect, ACS Nano, 2014, 8(12), 12866.

35 P. Gao, Z. Z. Wang, K. H. Liu, Z. Xu, W. L. Wang, X. D. Bai and E. G. Wang, Photoconducting response on bending of individual ZnO nanowires, J. Mater. Chem., 2009, 19(7), 1002-1005.

36 D. Somvanshi, A. Pandey and S. Jit, Ultraviolet Detection Characteristics of $\mathrm{Pd} / n-\mathrm{ZnO}$ Thin Film Schottky Photodiodes Grown on $n$-Si Substrates, J. Nanoelectron. Optoelectron., 2013, 8(4), 349-354.

37 M. M. H. Farooqi and R. K. Srivastava, Enhanced UV-vis photoconductivity and photoluminescence by doping of samarium in ZnO nanostructures synthesized by solid state reaction method, Optik, 2016, 127(8), 3991-3998.

38 Y. Liu, Y. Zhang, Q. Yang, S. Niu and Z. L. Wang, Fundamental theories of piezotronics and piezophototronics, Nano Energy, 2015, 14, 257-275. 